\title{
Use of extracorporeal membrane oxygenation for heart graft dysfunction in adults: incidence, risk factors and outcomes in a multicentric study
}

\author{
Pierre-Emmanuel Noly, MD, PhD \\ Mélanie Hébert, MD, MSc \\ Yoan Lamarche, MD, MSc \\ Jorge Robles Cortes \\ Marion Mauduit, MD \\ Jean-Philippe Verhoye, MD, PhD \\ Pierre Voisine, MD \\ Erwan Flécher, MD, PhD* \\ Michel Carrier, MD, MBA*
}

*These authors contributed equally to this
work.

Accepted Nov. 30, 2020

\section{Correspondence to: \\ P.E. Noly \\ Department of Cardiac Surgery \\ Université de Montréal \\ Montreal Heart Institute \\ 5000 rue Bélanger \\ Montréal QC H1T 1C8 \\ noly.pierreemmanuel@gmail.com}

Cite as: Can J Surg 2021 November 2; 64(6). doi: 10.1503/cjs.021319

\begin{abstract}
Background: The decision about whether to use venoarterial extracorporeal membrane oxygenation (VA-ECMO) in patients with cardiac graft dysfunction (GD) is usually made on a case-by-case basis and is guided by the team's experience. We aimed to determine the incidence of VA-ECMO use after heart transplantation (HT), to assess early- and long-term outcomes and to assess risk factors for the need for VA-ECMO and early mortality in these patients.
\end{abstract}

Methods: We included adults who underwent heart transplantation at 3 cardiac centres who met the most recent International Society for Heart and Lung Transplantation definition of graft dysfunction (GD) over a 10-year period. Pretransplant, intraoperative and posttransplant characteristics of the heart recipients as well as donor characteristics were analyzed and compared among recipients with GD treated with and without VA-ECMO.

Results: There were 135 patients with GD in this study, of whom 66 were treated with VA-ECMO and 69 were not. The mean follow-up averaged 81.2 months (standard deviation $36 \mathrm{mo}$, range $0-184 \mathrm{mo}$ ); follow-up was complete in $100 \%$ of patients. The overall incidence of GD (30\%) and of VA-ECMO use increased over the study period. We did not identify any predictive pretransplantation factors for VA-ECMO use, but patients who required VA-ECMO had higher serum lactate levels and higher inotropes doses after HT. The overall survival rates were $83 \%$ and $42 \%$ at 1 year and $78 \%$ and $40 \%$ at 5 years among patients who received only medical treatment and those who received VA-ECMO, respectively. Delayed initiation of VA-ECMO and postoperative bleeding were strongly associated with increased in-hospital mortality.

Conclusion: The incidence of GD increased over the study period, and the need for VA-ECMO among patients with GD remains difficult to predict. In-hospital mortality decreased over time but remained high among patients who required VA-ECMO, especially among patients with delayed initiation of VA-ECMO.

Contexte : La décision d'utiliser l'oxygénation extracorporelle (ECMO, pour extracorporeal membrane oxygenation) veino-artérielle chez les patients qui présentent une dysfonction du greffon (DG) cardiaque se prend habituellement au cas par cas et est guidée par l'expérience de l'équipe. Nous avons voulu déterminer l'incidence de l'utilisation de l'ECMO après une transplantation cardiaque (TC) afin d'en évaluer les résultats à court et à long terme, d'identifier les facteurs de risque de recours à l'ECMO et de mesurer la mortalité précoce chez ces patients.

Méthodes : Nous avons inclus des adultes qui avaient subi une transplantation cardiaque dans 3 centres de cardiologie et qui répondaient à la plus récente définition de dysfonction du greffon (DG) cardiaque de l'International Society for Heart and Lung Transplantation sur une période de 10 ans. Nous avons analysé les caractéristiques pré-, per- et post-transplantation des receveurs et les caractéristiques des donneurs et nous les avons comparées chez les receveurs ayant manifesté une DG, traités ou non par ECMO.

Résultats : Nous avons dénombré 135 patients ayant présenté une DG au cours de cette étude; 66 ont été traités par ECMO, contre 69 qui ne l'ont pas été. Le suivi moyen était de 81,2 mois (écart-type 36 mois, plage $0-184$ mois); le suivi a été mené à terme chez $100 \%$ des patients. L'incidence globale de la DG $(30 \%)$ et du recours à l'ECMO ont augmenté au cours de la période de l'étude. Nous n'avons pas identifié de facteurs prédictifs pré-transplantation pour ce qui est du recours à l'ECMO, mais les patients qui l'ont utilisée présentaient des taux plus élevés de lactase sérique et avaient reçu des doses d'inotropes plus élevés après la transplantation. Les taux de survie globaux ont été de $83 \%$ et de $42 \%$ à 1 an et de $78 \%$ et $40 \%$ à 5 ans chez les 
patients qui n'ont reçu que le traitement médical et qui ont reçu l'ECMO, respectivement. L'instauration retardée de l'ECMO et les saignements post-opératoires ont été étroitement associés à une mortalité perhospitalière accrue.

Conclusion : L'incidence de la DG a augmenté au cours de période de l'étude et le recours à l'ECMO veino-artérielle chez les patients présentant une DG reste difficile à prédire. La mortalité perhospitalière a diminué avec le temps, mais est demeurée élevée chez les patients qui ont eu besoin de l'ECMO, surtout si son instauration avait été retardé.

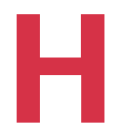

eart transplantation (HT) remains the treatment of choice for patients with end-stage heart failure refractory to optimal medical therapy. Donor shortages and increasing wait times for transplantation have driven teams to increase the use of marginal hearts. ${ }^{1}$ According to the 34th report of the International Society for Heart and Lung Transplantation (ISHLT), donor age and allograft ischemic time, both of which are risk factors for heart graft dysfunction (GD), have increased over the last 10 years. Despite many efforts to improve the medical management of patients after HT, $2.4 \%$ to $28 \%$ of heart recipients experience moderate to severe GD according to different studies and definitions, and it remains the leading cause of early mortality. ${ }^{2}$

Although management of severe primary graft failure varies among institutions, ${ }^{2}$ venoarterial extracorporeal membrane oxygenation (VA-ECMO) may improve the survival of patients with severe GD and high inotrope requirements. ${ }^{3-5}$ VA-ECMO allows the heart to recover by providing full hemodynamic support, systemic perfusion and oxygenation, and end-organ function recovery. Because it can be initiated rapidly, VA-ECMO is the first choice for temporary circulatory support in many institutions. ${ }^{3,4,6-8}$ However, data on the indications for VA-ECMO use after HT, the incidence of its use, and its short- and long-term impact on survival are still limited. Furthermore, the optimal timing of VA-ECMO initiation in severe GD is not fully established and remains mostly based on clinical judgment. A few studies have been reported in this population with a limited number of patients, but they all suggest that early initiation of mechanical support is associated with better survival. ${ }^{6,910}$ In this study, we aimed to determine predictive risk factors for the need for mechanical circulatory support with VA-ECMO in patients with GD after HT. We also aimed to determine outcomes and prognostic factors associated with mortality in these patients.

\section{Methods}

\section{Study population and data collection}

We retrospectively reviewed consecutive patients who underwent isolated orthotopic HT between January 2003 and December 2013 in 3 cardiac surgery centres: Rennes University Hospital (Rennes, France), the Quebec Heart and Lung Institute (IUCPQ, Quebec City, Quebec, Canada) and the Montreal Heart Institute (MHI, Montréal,
Quebec, Canada). This study was approved by the institutional review boards of the participating hospitals.

To reduce confounding factors, we excluded patients supported by VA-ECMO before and at the time of HT, patients who had emergent transplantation because of left ventricular assist device (LVAD) complications, patients who underwent combined heart and kidney transplantation and patients who had previous HT. We included in this study patients who had GD according to the most recent ISHLT definition. ${ }^{2}$ Patients who received VA-ECMO were divided into 2 subgroups according to the timing between $\mathrm{HT}$ and initiation of VA-ECMO: VA-ECMO was initiated within 24 hours of HT in the early-ECMO subgroup and more than 24 hours after HT in the delayed-ECMO subgroup.

Baseline demographic characteristics, medical history, laboratory values, hemodynamic values and echocardiographic data before HT were collected. Other data including intraoperative variables, donor characteristics and early and late post-transplant outcomes were also reviewed. For patients who required VA-ECMO, we reviewed the time between HT and initiation of VA-ECMO, and the doses of catecholamine and the levels of serum lactate at the time of VA-EMCO initiation. All data were obtained from electronic medical records, from patient charts, from local digital database systems and from the database of the Agence de la biomédecine in France (Cristal database).

\section{Definition of graft dysfunction}

Graft dysfunction was retrospectively defined according to the 2014 ISHLT consensus. ${ }^{2}$ Briefly, we considered GD when the following conditions were noted within 24 hours after HT: (1) right ventricle (RV), left ventricle (LV) or biventricular moderate to severe dysfunction on echocardiography (i.e., left ventricular ejection fraction [LVEF] < 40\%); (2) severe hemodynamic compromise (i.e., cardiac index $<2.0 \mathrm{~L} / \mathrm{min} / \mathrm{m}^{2}$ and high right and left atrial pressures) requiring prolonged high-dose inotropes (i.e., milrinone $>0.5 \mu \mathrm{g} / \mathrm{kg} / \mathrm{min}$, dobutamine $>0.5 \mu \mathrm{g} / \mathrm{kg} / \mathrm{min}$, adrenaline $>0.07 \mu \mathrm{g} / \mathrm{kg} / \mathrm{min}$, noradrenaline $>0.7 \mu \mathrm{g} / \mathrm{kg} / \mathrm{min})^{11}$ or nitric oxide; (3) need for mechanical circulatory support; (4) inability to wean the patient off cardiopulmonary bypass, or difficulty doing so, despite adequate reperfusion; or (5) use of intra-aortic balloon pump. We included all patients with moderate to severe primary or secondary GD. Heart transplant procedures and recipient management are described in Appendix 1 (available at canjsurg.ca). 


\section{Statistical analysis}

Continuous variables are presented as means with standard deviations (SDs) and categorical variables as percentages. Univariate analysis was performed using $\chi^{2}$ tests for categorical variables, unpaired $t$ tests for normally distributed continuous variables after verifying for equality of variances, and Mann-Whitney $U$ tests when equality of variances was not respected. A stepwise logistic regression with a cut-off of $p=0.20$ was performed to determine predictors of the need for VA-ECMO support among patients with GD. To measure model discrimination, C statistics (area under the receiver operating characteristic curve) were used, and the Hosmer-Lemeshow test was used to assess goodness of fit. Early outcomes, causes of GD, complications and survival after HT were compared between patients supported with VA-ECMO and patients who were treated medically. Actuarial survival of patients is presented using Kaplan-Meier curves with $p$ values calculated using $\log$-rank tests. A 2 -sided $p<0.05$ was considered to be statistically significant.

Weaning off VA-ECMO, duration of support, rates and causes of deaths, and complications while on VA-ECMO were compared between the early- and delayed-ECMO subgroups. The complications that were included were ischemic and vascular complications, bleeding, stroke, need for reoperation, renal status and organ rejection.

To assess the effect of time on VA-EMCO use and outcomes, we divided the 10 -year study period into 3 periods: period 1 (2003-2006), period 2 (2007-2010) and period 3 (2011-2013). Mann-Kendall trend tests and one-way analysis of variance were used to detect differences between periods. Outcomes were also compared between patients who had early versus delayed VA-ECMO support. Statistical analyses were performed using GraphPad (Prism 6, GraphPad Software) and SPSS Statistics for MAC, version 22.0. (IBM Corp.).

\section{RESULtS}

\section{Patient characteristics and risk factors for VA-ECMO in patients with graft dysfunction}

A total of 454 consecutive patients underwent isolated orthotopic HT in the 3 study centres between January 2003 and December 2013: 161 at the Rennes University Hospital 158 at the IUCPQ, and 135 at the MHI. Of these patients, we excluded 34 patients who were

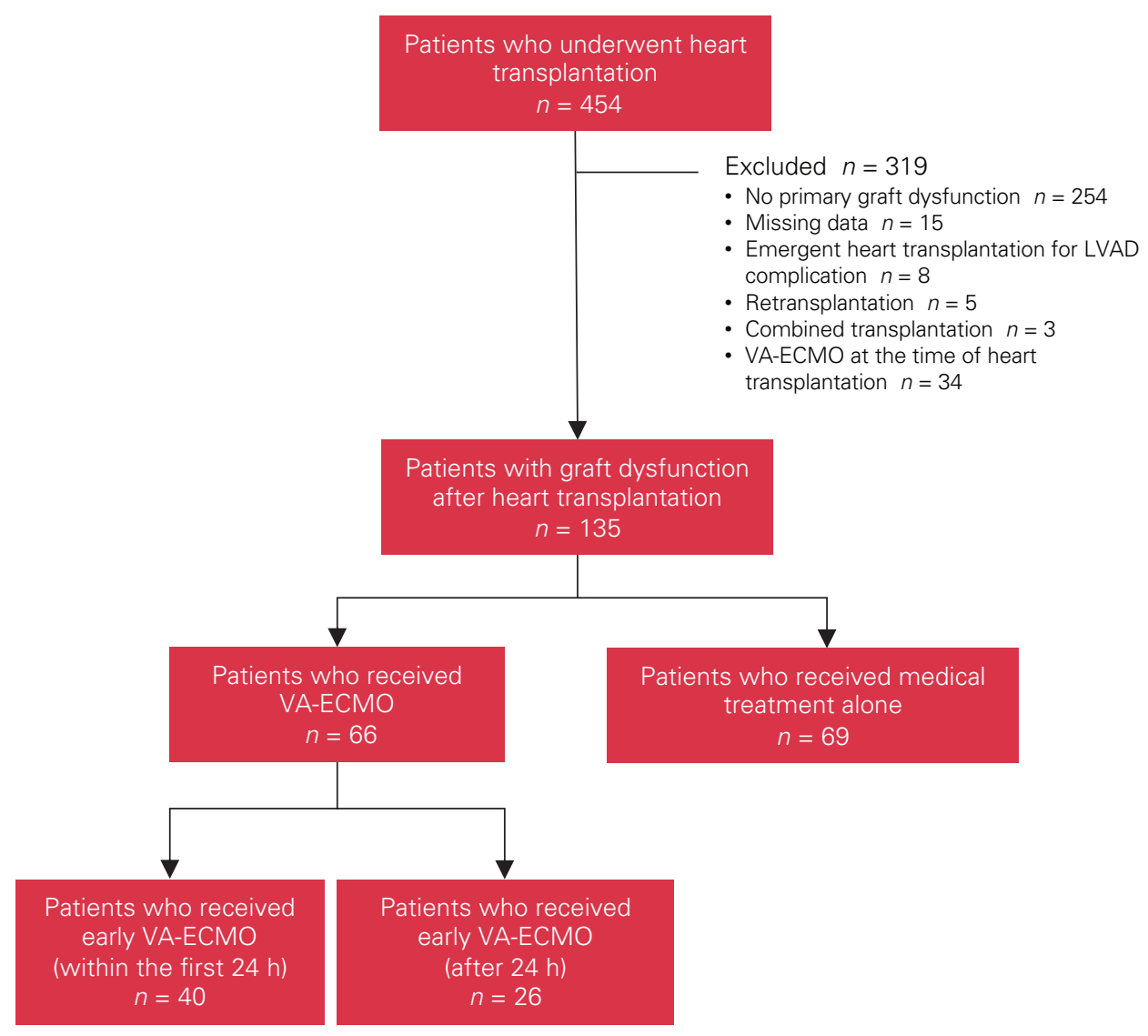

Fig. 1. Study flowchart. LVAD = left ventricular assist device; VA-ECMO = venoarterial extracorporeal membrane oxygenation. 
supported by VA-ECMO before and at the time of HT, 8 who had emergent transplantation because of LVAD complications, 3 who underwent combined heart and kidney transplantation and 5 who had previous HT.

We included in this study the 135 patients $(30 \%)$ who had GD according to the most recent ISHLT definition (Figure 1). ${ }^{2}$ Of these, $106(78 \%)$ presented with primary GD and 29 (22\%) had GD secondary to pulmonary hypertension. Seventy (66\%) of them had RV failure, 24 (22\%) had biventricular failure and 12 (11\%) had LV failure (Figure 2).

Sixty-six (49\%) of the 135 study patients required mechanical circulatory support with VA-ECMO, and 69 $(51 \%)$ patients were treated medically (Figure 1). Patients in the VA-ECMO group were more likely to have biventricular failure. Twenty-nine (22\%) patients had RV failure secondary to pulmonary hypertension, which did not differ between patients treated with or without VA-ECMO. In the VA-ECMO group, the average time between the end of HT and the start of VA-ECMO support was 27.3 (SD 48.2) hours. In the early-ECMO subgroup $(n=40,61 \%)$, the mean time to VA-ECMO initiation was 3.2 (SD 2.2) hours. In the delayed-ECMO subgroup $(n=26,39 \%)$, the mean time to VA-ECMO initiation was 38.2 (SD 10) hours. In 26 (39\%) patients, VA-ECMO was initiated more than 24 hours after HT. Fifty-three (80\%) patients had a peripheral cannulation, 17 (25\%) had a central cannulation and $4(6 \%)$ patients needed conversion from a peripheral to a central cannulation because of limb ischemia.

Donor and recipient characteristics before HT did not differ significantly among the institutions (data not shown). Follow-up was conducted until December 2018 and averaged 81.2 months (SD 36 mo, range 0-184 mo). Clinical follow-up was complete in $100 \%$ of patients.

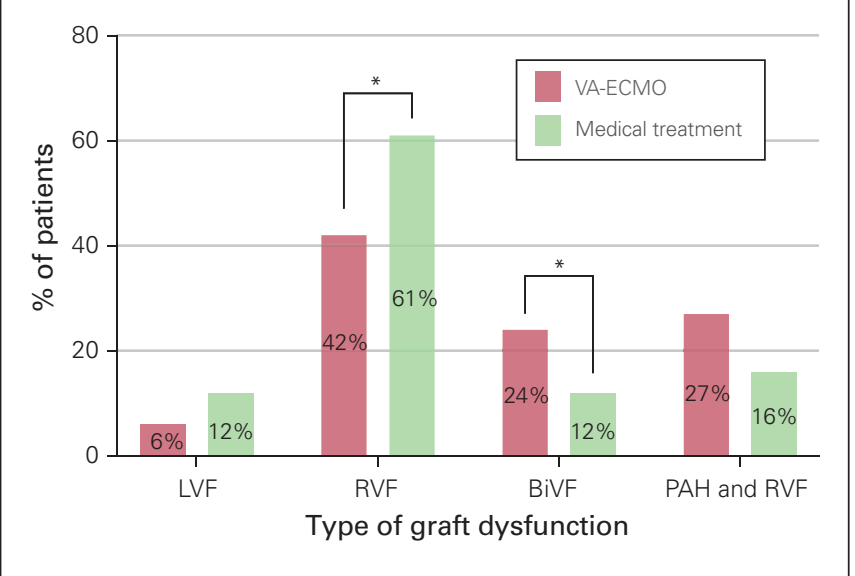

Fig. 2. Distribution of the types of graft dysfunction among patients who received $V A-E C M O$ and those who received medical treatment. BiVF = biventricular failure; LVF = left ventricular failure; $\mathrm{PAH}=$ pulmonary arterial hypertension; $\mathrm{RVF}=$ right ventricular failure; VA-ECMO = venoarterial extracorporeal membrane oxygenation. ${ }^{*} p<0.05$.
Pretransplantation patient characteristics and donor characteristics are summarized in Table 1 and Table 2. We did not observe any differences between the patients who received VA-EMCO and those who did not in terms of demographic characteristics, comorbidities, cause of cardiomyopathy, clinical status at admission, hemodynamics, echocardiographic data and biologic characteristics. The donor characteristics, the ischemia time and the recipient-donor match characteristics were also similar. We noticed that patients who required mechanical circulatory support had higher doses of adrenaline $(0.59$ [SD 0.08] v. 0.27 [SD 0.02] $\mu \mathrm{g} / \mathrm{kg} / \mathrm{min}, p=0.001)$ and noradrenaline $(0.83$ [SD 1.9] v. 0.10 [SD 0.33$] \mu \mathrm{g} / \mathrm{kg} / \mathrm{min}$, $p=0.001)$ and had a higher peak of serum lactates (14.5 [SD 6.4] v. $8.2[\mathrm{SD} 3.9] \mathrm{mmol} / \mathrm{L}, p=0.05$ ) within the first hours after transplantation.

We did not find any pretransplant or donor-related predictive factors for the use of VA-ECMO (Appendix 1, Table 1S). However, the peak of serum lactates after transplantation was significantly associated with the use of VAECMO in univariate and multivariate analyses (odds ratio [OR] 1.29, 95\% confidence interval [CI] 1.06-1.8, $p=0.01$ ).

\section{Outcomes after graft dysfunction and long-term survival}

In-hospital outcomes are summarized in Table 3 . Inhospital mortality was $36 \%(n=48)$ in the entire cohort. It was significantly higher in patients requiring VA-ECMO ( $57 \%$ v. $14 \%, p=0.001)$; however, the length of stay in the intensive care unit and in the hospital did not differ between the 2 groups. Reoperation for bleeding and acute kidney injury including the use of temporary dialysis were the most frequent complications, and they occurred significantly more frequently in patients requiring VA-ECMO than in those who did not. The rates of other complications such as stroke and acute rejection did not significantly differ between the groups. Complications related to VA-ECMO were as follows: need for any blood product transfusion (100\%), reoperation for bleeding (64\%), cannula site complications (sepsis, lymphocele) (21\%) and limb ischemia (12\%) (Table 4 and Appendix 1, Table 3S).

The overall survival in the entire cohort (KaplanMeier curves) was $63 \%, 59 \%$ and $52 \%$ at 1, 5 and 10 years, respectively. It was significantly lower in patients who required VA-ECMO (42\% v. $83 \%$ at $1 \mathrm{yr}$ and $40 \%$ v. $78 \%$ at $5 \mathrm{yr}$ ) (Figure $3 \mathrm{~A}$ ).

\section{Risk factors for increased mortality after graft dysfunction in patients receiving VA-ECMO}

In the VA-EMCO group, patients who did not survive until hospital discharge were more likely to have had an emergent HT ( $p=0.03)$, a shorter wait $(p=0.05)$ and cardiac arrest as the indication for VA-ECMO $(p=0.05)$ 
Table 1. Pretransplantation characteristics of study patients

\begin{tabular}{|c|c|c|c|c|}
\hline \multirow[b]{2}{*}{ Characteristic } & \multicolumn{3}{|c|}{ No. $(\%)$ of patients* } & \multirow[b]{2}{*}{$p$ value } \\
\hline & $\begin{array}{c}\text { All } \\
n=135\end{array}$ & $\begin{array}{l}\text { With VA-ECMO } \\
\qquad n=66\end{array}$ & $\begin{array}{l}\text { Without VA-ECMO } \\
\qquad n=69\end{array}$ & \\
\hline \multicolumn{5}{|l|}{ Demographic characteristics } \\
\hline Age, $y r$, mean $\pm S D$ & $50 \pm 11$ & $49 \pm 12$ & $52 \pm 10$ & 0.08 \\
\hline Age $>60 \mathrm{yr}$ & $36(27)$ & $16(24)$ & $20(29)$ & 0.53 \\
\hline Male sex & $39(29)$ & $15(28)$ & $24(35)$ & 0.12 \\
\hline Weight, $\mathrm{kg}$, mean $\pm \mathrm{SD}$ & $74 \pm 14$ & $74 \pm 13$ & $73 \pm 15$ & 0.66 \\
\hline \multicolumn{5}{|l|}{ Medical history } \\
\hline Diabetes with insulin & $13(10)$ & $5(8)$ & $8(12)$ & 0.41 \\
\hline Hypertension & $34(25)$ & $15(23)$ & $19(27)$ & 0.52 \\
\hline History of tobacco use & $43(32)$ & $23(35)$ & $20(29)$ & 0.50 \\
\hline Atrial fibrillation & $66(49)$ & $33(50)$ & $33(49)$ & 0.86 \\
\hline $\begin{array}{l}\text { Chronic obstructive pulmonary disease (FEV1 } \\
<70 \%)\end{array}$ & $13(10)$ & $6(9)$ & $7(10)$ & 0.81 \\
\hline Previous stroke & $19(14)$ & $10(15)$ & $9(13)$ & 0.75 \\
\hline $\begin{array}{l}\text { Chronic kidney failure (GFR }<60 \mathrm{~mL} / \mathrm{min} \text { in } \\
\text { MDRD) }\end{array}$ & $44(33)$ & $19(29)$ & $25(36)$ & 0.35 \\
\hline Previous sternotomy & $43(32)$ & $24(36)$ & $19(28)$ & 0.29 \\
\hline $\begin{array}{l}\text { Highly sensitized (Panel reactive antibody } \\
>80 \%)\end{array}$ & $17(13)$ & $11(17)$ & $6(9)$ & 0.16 \\
\hline Left ventricular assist device (HeartMate II) & $43(32)$ & $23(35)$ & $20(29)$ & 0.50 \\
\hline \multicolumn{5}{|l|}{ Cause of cardiomyopathy } \\
\hline Ischemic & $44(33)$ & $24(36)$ & $20(29)$ & 0.36 \\
\hline Dilated nonischemic or idiopathic & $39(29)$ & $17(26)$ & $22(32)$ & 0.43 \\
\hline Hypertrophic & $21(16)$ & $11(17)$ & $10(14)$ & 0.72 \\
\hline Valvular & $14(10)$ & $9(14)$ & $5(7)$ & 0.22 \\
\hline Congenital & $9(7)$ & $6(9)$ & $3(4)$ & 0.26 \\
\hline Other & $23(17)$ & $11(17)$ & $12(17)$ & 0.91 \\
\hline \multicolumn{5}{|l|}{ Clinical status at the time of heart transplantation } \\
\hline In ICU at the time of transplantation & $31(23)$ & $17(26)$ & $14(20)$ & 0.53 \\
\hline $\begin{array}{l}\text { Waiting time for heart transplantation, } d \\
\text { mean } \pm S D\end{array}$ & $174 \pm 266$ & $169 \pm 219$ & $179 \pm 306$ & 0.83 \\
\hline Acute kidney failure (GFR $<40 \mathrm{~mL} / \mathrm{min}$ in MDRD) & $29(22)$ & $12(18)$ & $17(25)$ & 0.36 \\
\hline Acute liver failure (bilirubin $>30 \mu \mathrm{mol} / \mathrm{L}$ ) & $21(16)$ & $11(17)$ & $10(15)$ & 0.53 \\
\hline Low cardiac output syndrome & $52(38)$ & $23(34)$ & $29(44)$ & 0.31 \\
\hline Mechanical ventilation before heart transplantation & $4(3)$ & $2(3)$ & $2(3)$ & 0.96 \\
\hline Intra-aortic balloon pump & $18(13)$ & $7(11)$ & $11(16)$ & 0.34 \\
\hline Inotropic support (any catecholamine) & $36(27)$ & $15(23)$ & $21(30)$ & 0.31 \\
\hline \multicolumn{5}{|l|}{ Echocardiographic characteristic } \\
\hline Left ventricular ejection fraction, $\%$, mean \pm SD & $10.5 \pm 17$ & $9.0 \pm 18$ & $11.9 \pm 16.1$ & 0.34 \\
\hline \multicolumn{5}{|l|}{ Hemodynamic characteristics } \\
\hline Cardiac index, $\mathrm{L} /$ min per $\mathrm{m}^{2}$, mean $\pm \mathrm{SD}$ & $2.0 \pm 0.5$ & $2.1 \pm 0.6$ & $2.0 \pm 0.6$ & 0.61 \\
\hline $\begin{array}{l}\text { Pulmonary vascular resistance, Wood units, } \\
\text { mean } \pm \text { SD }\end{array}$ & $2.3 \pm 1.2$ & $2.4 \pm 1.3$ & $2.3 \pm 1.2$ & 0.86 \\
\hline $\begin{array}{l}\text { Systolic pulmonary arterial pressure, } \mathrm{mm} \mathrm{Hg} \\
\text { mean } \pm \mathrm{SD}\end{array}$ & $42 \pm 14$ & $43 \pm 15$ & $41 \pm 14$ & 0.60 \\
\hline $\begin{array}{l}\text { Mean pulmonary arterial pressure, } \mathrm{mm} \mathrm{Hg} \\
\text { mean } \pm \mathrm{SD}\end{array}$ & $29 \pm 10$ & $30 \pm 11$ & $28 \pm 10$ & 0.34 \\
\hline \multicolumn{5}{|l|}{ Biologic characteristics } \\
\hline Glomerular filtration rate, $\mathrm{mL} / \mathrm{min}$, mean $\pm \mathrm{SD}$ & $68 \pm 30$ & $66 \pm 26$ & $60 \pm 29$ & 0.34 \\
\hline Total bilirubin, $\mathrm{mg} / \mathrm{dL}$, mean $\pm \mathrm{SD}$ & $17.6 \pm 10.2$ & $17.9 \pm 10.3$ & $17.9 \pm 10$ & 0.75 \\
\hline $\mathrm{AST}, \mathrm{IU} / \mathrm{L}$, mean $\pm \mathrm{SD}$ & $45.5 \pm 52.1$ & $45.8 \pm 49.9$ & $45.2 \pm 55.6$ & 0.94 \\
\hline $\mathrm{ALT}, \mathrm{IU} / \mathrm{L}$, mean $\pm \mathrm{SD}$ & $44.8 \pm 40$ & $52.4 \pm 51.5$ & $37.4 \pm 23.9$ & 0.03 \\
\hline MELD-XI score, mean \pm SD & $11.8 \pm 4.8$ & $11.7 \pm 4.4$ & $12.5 \pm 5.9$ & 0.66 \\
\hline \multicolumn{5}{|c|}{$\begin{array}{l}\text { ALT = alanine aminotransferase; } A S T=\text { aspartate aminotransferase; FEV1 }=\text { forced expiratory volume in } 1 \mathrm{~s} ; \mathrm{GFR}=\text { glomerular filtration rate; ICU = intensive care } \\
\text { unit; MDRD = Modification of Diet in Renal Disease study equation; MELD-XI = Model for End-stage Liver Disease score excluding INR; SD = standard deviation } \\
\text { VA-ECMO = venoarterial extracorporeal membrane oxygenation. } \\
\text { *Unless indicated otherwise. }\end{array}$} \\
\hline
\end{tabular}


Table 2. Donor characteristics, recipient-donor match and operative data

\begin{tabular}{|c|c|c|c|c|}
\hline \multirow[b]{2}{*}{ Measure } & \multicolumn{3}{|c|}{ No. $(\%)$ of patients* } & \multirow[b]{2}{*}{$p$ value } \\
\hline & $\begin{array}{c}\text { All } \\
n=135\end{array}$ & $\begin{array}{c}\text { With } \\
\text { VA-ECMO } \\
n=66\end{array}$ & $\begin{array}{c}\text { Without } \\
\text { VA-ECMO } \\
n=69\end{array}$ & \\
\hline \multicolumn{5}{|l|}{ Donor characteristics } \\
\hline Age, yr, mean $\pm S D$ & $40.7 \pm 13.4$ & $40.8 \pm 12$ & $41 \pm 14$ & 0.57 \\
\hline Age $>50 y r$ & $37(27)$ & $16(24)$ & $21(30)$ & 0.42 \\
\hline Male sex & $94(70)$ & $54(81)$ & $40(58)$ & 0.03 \\
\hline \multicolumn{5}{|l|}{ Cause of donor death } \\
\hline Trauma & $30(22)$ & $15(22)$ & $15(22)$ & 0.92 \\
\hline Intoxication & $4(3)$ & 2 (3) & 2 (3) & 0.97 \\
\hline Cerebral hemorrhage & $60(45)$ & $34(51)$ & $26(38)$ & 0.12 \\
\hline Ischemic stroke & $35(26)$ & $22(33)$ & $13(19)$ & 0.06 \\
\hline Death by hanging & $12(9)$ & $3(4)$ & $9(13)$ & 0.07 \\
\hline Cardiac arrest & $31(23)$ & $16(24)$ & $15(22)$ & 0.76 \\
\hline $\begin{array}{l}\text { Distance from recipient, } \mathrm{km}, \\
\text { mean } \pm \mathrm{SD}\end{array}$ & $308 \pm 383$ & $283 \pm 361$ & $332 \pm 405$ & 0.45 \\
\hline \multicolumn{5}{|l|}{ Recipient-donor match } \\
\hline Mismatch weight > 20\% & $34(25)$ & $18(27)$ & $16(23)$ & 0.58 \\
\hline \multicolumn{5}{|l|}{ Mismatch sex } \\
\hline Recipient man, donor woman & $16(12)$ & $6(9)$ & $10(14)$ & 0.33 \\
\hline Recipient woman, donor man & $14(10)$ & $9(14)$ & $5(7)$ & 0.22 \\
\hline RADIAL score, mean \pm SD & $1.8 \pm 0.9$ & $1.7 \pm 0.9$ & $1.9 \pm 1.0$ & 0.32 \\
\hline \multicolumn{5}{|l|}{ Operative data } \\
\hline $\begin{array}{l}\text { Graft ischemia time, min, } \\
\text { mean } \pm \text { SD }\end{array}$ & $194 \pm 63$ & $200 \pm 68$ & $188 \pm 58$ & 0.24 \\
\hline Graft ischemia time $>240 \mathrm{~min}$ & $32(23)$ & $19(28)$ & $13(19)$ & 0.17 \\
\hline $\begin{array}{l}\text { Cardiopulmonary bypass time, } \\
\text { min, mean } \pm \text { SD }\end{array}$ & $148 \pm 61$ & $171 \pm 62$ & $126 \pm 53$ & 0.07 \\
\hline $\begin{array}{l}\text { RADIAL = right atrial pressure } \geq 10 \mathrm{~mm} \\
\text { time } \geq 240 \mathrm{~min} ; \mathrm{SD}=\text { standard deviation; } \\
{ }^{*} \text { Unless indicated otherwise. }\end{array}$ & $\begin{array}{l}\text { age } \geq 60 \mathrm{yr} \\
\text { venoarteria }\end{array}$ & membran & onor age & schemic \\
\hline
\end{tabular}

Table 3. In-hospital outcomes after heart transplantation

\begin{tabular}{|c|c|c|c|c|}
\hline \multirow[b]{2}{*}{ Measure } & \multicolumn{3}{|c|}{ No. (\%) of patients* } & \multirow[b]{2}{*}{$p$ value } \\
\hline & $\begin{array}{c}\text { All } \\
n=135\end{array}$ & $\begin{array}{l}\text { With } \\
\text { VA-ECMO } \\
n=66\end{array}$ & $\begin{array}{l}\text { Without VA-ECMO } \\
\qquad n=69\end{array}$ & \\
\hline In-hospital mortality & $48(36)$ & $38(57)$ & $10(14)$ & 0.001 \\
\hline LOS in ICU, d, mean $\pm S D$ & $10 \pm 11$ & $12 \pm 15$ & $9.2 \pm 8$ & 0.10 \\
\hline LOS in hospital, d, mean \pm SD & $24 \pm 31$ & $29 \pm 43$ & $20 \pm 15$ & 0.15 \\
\hline $\begin{array}{l}\text { Bleeding during the first } 24 \mathrm{~h} \text {, } \\
\mathrm{mL} \text {, mean } \pm \mathrm{SD}\end{array}$ & $1523 \pm 789$ & $1906 \pm 257$ & $1238 \pm 222$ & 0.02 \\
\hline Any transfusions & $124(93)$ & $66(100)$ & $60(88)$ & 0.005 \\
\hline \multicolumn{5}{|l|}{ Other complications } \\
\hline Acute kidney injury & $89(68)$ & $53(83)$ & $36(54)$ & 0.001 \\
\hline Temporary CRRT & $50(38)$ & $31(50)$ & $19(28)$ & 0.01 \\
\hline $\begin{array}{l}\text { Maximal level of creatinine, } \\
\mathrm{mmol} / \mathrm{L} \text {, mean } \pm \mathrm{SD}\end{array}$ & $255 \pm 127$ & $286 \pm 137$ & $224 \pm 109$ & 0.005 \\
\hline $\begin{array}{l}\text { Minimal glomerular filtration } \\
\text { rate, } \mathrm{mL} / \mathrm{min} \text {, mean } \pm \mathrm{SD}\end{array}$ & $35.7 \pm 19.9$ & $32.4 \pm 17$ & $39.3 \pm 21$ & 0.03 \\
\hline Stroke & $3(3)$ & 2 (4) & $1(2)$ & 0.46 \\
\hline $\begin{array}{l}\text { Acute rejection } \\
\text { (ISHLT grade } 2 \mathrm{R} \text { or }+ \text { ) }\end{array}$ & $19(16)$ & $9(18)$ & $10(15)$ & 0.70 \\
\hline Reoperation for bleeding & $58(43)$ & $42(64)$ & $16(23)$ & 0.001 \\
\hline \multicolumn{5}{|c|}{$\begin{array}{l}\text { CRRT = continuous renal replacement therapy; ICU = intensive care unit; ISHLT = International Society for Heart and Lung Transplantation; } \\
\text { LOS = length of stay; } \mathrm{SD}=\text { standard deviation; VA-ECMO = venoarterial extracorporeal membrane oxygenation. } \\
\text { *Unless indicated otherwise. }\end{array}$} \\
\hline
\end{tabular}


A

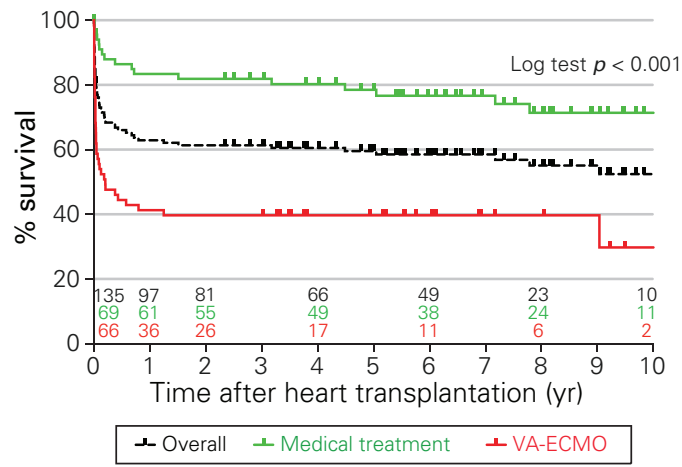

C

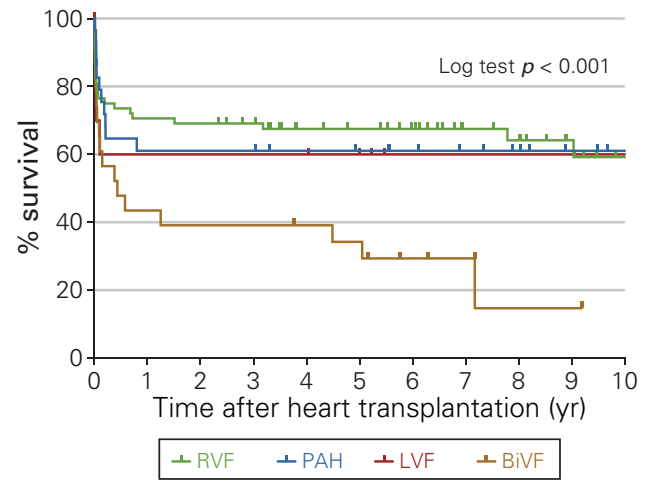

B

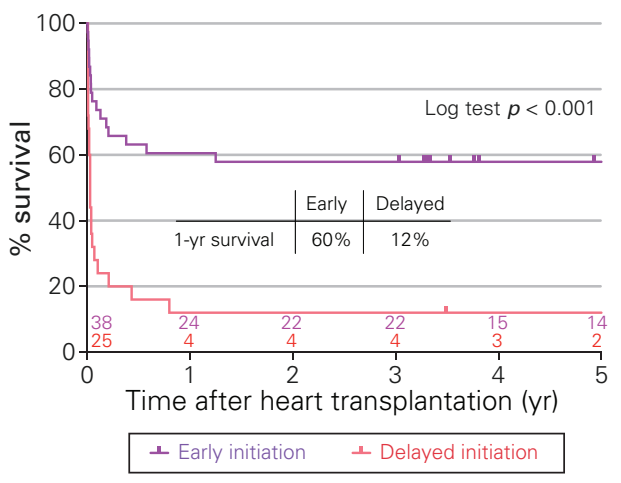

D

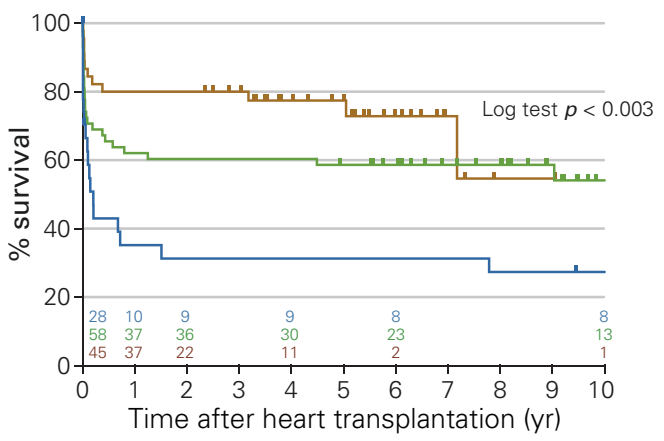

LPeriod 1 (2003-2006) +Period 2 (2007-2010) -Period 3 (2011-2013)

Fig. 3. (A) Overall survival in the study cohort and survival after graft dysfunction treated medically or with VA-ECMO. (B) Survival assessed according to the delay in VA-ECMO initiation after heart transplantation. (C) Survival according to the classification of the graft dysfunction. (D) Survival according to the study period. BiVF = biventricular failure; $\mathrm{LVF}=$ left ventricular failure; $\mathrm{PAH}=$ pulmonary arterial hypertension; RVF = right ventricular failure; $\mathrm{VA}-\mathrm{ECMO}=\mathrm{veno}-$ arterial extracorporeal membrane oxygenation.

(Table 4). Likewise, indicators of organ failure were also significantly higher in patients who died, such as acute liver failure $(p=0.01)$, low cardiac output syndrome $(p=0.05)$ and lower mean pulmonary arterial pressure $(p=0.008)$. The rates of delayed initiation of VA-ECMO and postoperative bleeding were significantly higher in patients who $\operatorname{died}(2350[\mathrm{SD} 270 \mathrm{~mL}]$ v. 1279 [SD $944 \mathrm{~mL}], p=0.03)$.

In multivariate analysis for in-hospital mortality (Table 5), only delayed initiation of VA-ECMO (OR 5.11, 95\% CI $1.08-24.22, p=0.04$ ) and 24-hour bleeding (OR 1.00, 95\% CI $1.00-1.01, p=0.05$ ) were significantly associated with increased in-hospital mortality. Survival was very poor in the delayed-ECMO subgroup (12\% v. $60 \%$ at $1 \mathrm{yr}, p<0.001)$ (Figure 3B). Interestingly, pretransplant characteristics including demographic characteristics, comorbidities, biologic parameters, indication for ECMO and recipient-donor match were similar between the delayed-ECMO and earlyECMO subgroups (Appendix 1, Table 2S). Furthermore, there were no significant differences in organ failure indicators, such as incidence of acute kidney failure $(p=0.74)$, acute liver failure $(p=0.99)$, Model for End-stage Liver Disease score excluding INR (MELD-XI) ( $p=0.65)$, inotrope duration $(p=0.29)$ and maximum noradrenaline $(p=0.32)$ and adrenaline $(p=0.48)$ doses. However, the peak of serum lactates before VA-ECMO initiation was higher in the delayedECMO subgroup (18.0 [SD 6] mmol/L v. 11.6 [SD 5] $\mathrm{mmol} / \mathrm{L}, p=0.005)$, suggesting a more advanced clinical state before initiation of mechanical circulatory support. The type of GD was not associated with increased mortality, but patients with a biventricular failure had a lower survival rate than the others (Figure 3C).

\section{Incidence of graft dysfunction, use of VA-ECMO and mortality over the study period}

There were 29 patients in period 1 (2003-2006), 59 in period 2 (2007-2010) and 47 in period 3 (2011-2013). We observed an increase in the incidence of GD (treated medically and with VA-ECMO) during the study period from $23 \%$ in period 1 to $41 \%$ in period $3(p=0.001)$ (Figure 4A). The observed changes in recipient characteristics over time were an increase in glomerular 
Table 4. Comparison between survivors and nonsurvivors among patients who received VA-ECMO

No. (\%) of patients*

\begin{tabular}{|c|c|c|c|}
\hline Characteristic & $\begin{array}{c}\text { Survivors } \\
n=28\end{array}$ & $\begin{array}{c}\text { Nonsurvivors } \\
n=38\end{array}$ & $p$ value \\
\hline \multicolumn{4}{|l|}{ Pretransplantation characteristics } \\
\hline Age, yr, mean \pm SD & $49 \pm 13$ & $48 \pm 12$ & 0.65 \\
\hline Emergent heart transplantation & $5(18)$ & $16(42)$ & 0.03 \\
\hline Highly sensitized (panel reactive antibody > 80\%) & $6(21)$ & $5(13)$ & 0.37 \\
\hline Chronic kidney failure (GFR $<60 \mathrm{~mL} / \mathrm{min}$ in MDRD) & $7(25)$ & $12(32)$ & 0.56 \\
\hline Previous sternotomy & $10(35)$ & $14(37)$ & 0.92 \\
\hline In ICU at the time of transplantation & $4(15)$ & $13(34)$ & 0.08 \\
\hline Waiting time, $\mathrm{d}$, mean $\pm \mathrm{SD}$ & $230 \pm 285$ & $124 \pm 142$ & 0.05 \\
\hline Acute kidney failure (GFR $<40 \mathrm{ml} / \mathrm{min}$ in MDRD) & $5(18)$ & $7(19)$ & 0.91 \\
\hline Acute liver failure (bilirubin $>30 \mu \mathrm{mol} / \mathrm{L}$ ) & $1(4)$ & $10(26)$ & 0.01 \\
\hline Low cardiac output syndrome & $6(22)$ & $17(46)$ & 0.05 \\
\hline Mean pulmonary arterial pressure, $\mathrm{mm} \mathrm{Hg}$, mean $\pm \mathrm{SD}$ & $34 \pm 10$ & $26 \pm 11$ & 0.008 \\
\hline $\mathrm{GFR}, \mathrm{mL} / \mathrm{min}$, mean $\pm \mathrm{SD}$ & $70 \pm 29$ & $63 \pm 23$ & 0.26 \\
\hline Pulmonary vascular resistance, Wood units, mean \pm SD & $2.38 \pm 1.1$ & $2.39 \pm 1.4$ & 0.97 \\
\hline Total bilirubin, $\mathrm{mg} / \mathrm{dL}$, mean $\pm \mathrm{SD}$ & $16 \pm 8$ & $19 \pm 11$ & 0.33 \\
\hline Inotropic support (any catecholamine) & $6(21)$ & $9(24)$ & 0.82 \\
\hline MELD-XI score, mean \pm SD & $10.1 \pm 4.0$ & $12.8 \pm 4.4$ & 0.16 \\
\hline RADIAL score, mean \pm SD & $1.6 \pm 0.9$ & $1.9 \pm 0.9$ & 0.31 \\
\hline Ischemia time, mean \pm SD & $199 \pm 62$ & $201 \pm 72$ & 0.92 \\
\hline \multicolumn{4}{|l|}{ Indications for ECMO (nonexclusive) } \\
\hline \multicolumn{4}{|l|}{ Primary graft dysfunction } \\
\hline Left ventricle & $4(14)$ & $4(10)$ & 0.64 \\
\hline Right ventricle & $13(46)$ & $20(53)$ & 0.61 \\
\hline Biventricular & $5(18)$ & $10(26)$ & 0.46 \\
\hline \multicolumn{4}{|l|}{ Secondary graft dysfunction } \\
\hline Right ventricle failure due to pulmonary hypertension & $10(36)$ & $10(26)$ & 0.41 \\
\hline Cardiac arrest & 0 & $8(35)$ & 0.05 \\
\hline \multicolumn{4}{|l|}{ Patient characteristics before VA-ECMO } \\
\hline Maximum noradrenaline dose, $\mu \mathrm{g} / \mathrm{kg}$ per min, mean $\pm \mathrm{SD}$ & $0.75 \pm 1.9$ & $0.87 \pm 1.8$ & 0.85 \\
\hline Maximum adrenaline dose, $\mu \mathrm{g} / \mathrm{kg}$ per min, mean $\pm \mathrm{SD}$ & $0.22 \pm 0.21$ & $0.48 \pm 0.6$ & 0.08 \\
\hline Inotrope duration, mean \pm SD & $15 \pm 16$ & $8 \pm 7$ & 0.06 \\
\hline Peak of serum lactates, $\mathrm{mmol} / \mathrm{L}$, mean $\pm \mathrm{SD}$ & $13 \pm 4$ & $15 \pm 6$ & 0.48 \\
\hline Delayed initiation of VA-ECMO & $4(14)$ & $22(58)$ & 0.001 \\
\hline \multicolumn{4}{|l|}{ Technical considerations } \\
\hline Peripheral & $27(96)$ & $26(68)$ & 0.005 \\
\hline Conversion from peripheral to central & $1(4)$ & $4(10)$ & 0.25 \\
\hline Central & $1(4)$ & $16(42)$ & 0.001 \\
\hline \multicolumn{4}{|l|}{ Complications under VA-ECMO } \\
\hline Reoperation for bleeding or tamponade & $17(60)$ & $25(67)$ & 0.56 \\
\hline Lower limb ischemia & $2(7)$ & $6(16)$ & 0.27 \\
\hline Bleeding requiring blood transfusions & $13(48)$ & $23(60)$ & 0.32 \\
\hline 24-h bleeding, mean \pm SD & $1279 \pm 944$ & $2350 \pm 270$ & 0.03 \\
\hline \multicolumn{4}{|l|}{ Recipient-donor match } \\
\hline Mismatch weight > 20\% & $9(32)$ & $9(23)$ & 0.44 \\
\hline \multicolumn{4}{|l|}{ Mismatch sex } \\
\hline Recipient man, donor woman & $2(7)$ & $4(10)$ & 0.63 \\
\hline Recipient woman, donor man & $4(14)$ & $5(13)$ & 0.89 \\
\hline \multicolumn{4}{|c|}{$\begin{array}{l}\text { GFR = glomerular filtration rate; ICU = intensive care unit; MDRD = Modification of Diet in Renal Disease study equation; MELD-XI = Model for End-stage Liv } \\
\text { Disease score excluding INR; RADIAL = right atrial pressure } \geq 10 \mathrm{~mm} \mathrm{Hg} \text {, recipient age } \geq 60 \mathrm{yr} \text {, diabetes mellitus, inotrope dependence, donor age } \geq 30 \mathrm{yr} \text {, } \\
\text { length of ischemic time } \geq 240 \mathrm{~min} ; \mathrm{SD}=\text { standard deviation; } \mathrm{VA}-\mathrm{ECMO}=\text { venoarterial extracorporeal membrane oxygenation. }\end{array}$} \\
\hline
\end{tabular}

filtration rate $(p=0.02)$, a decrease in insulin-treated diabetes $(p=0.01)$, a decrease in total bilirubin $(p=0.04)$, a decrease in use of intra-aortic balloon pump ( $p=0.007)$ and a decrease in MELD-XI score $(p=0.002)$. Also, the number of patients in the intensive care unit before HT decreased over time $(p=0.05)$. We also noticed that the 
Table 5. Univariate and multivariate analyses for hospital mortality in patients supported with VA-ECMO

\begin{tabular}{|c|c|c|c|c|c|c|}
\hline \multirow[b]{2}{*}{ Variable } & \multicolumn{3}{|c|}{ Univariate analysis } & \multicolumn{3}{|c|}{ Multivariate analysis } \\
\hline & OR & $95 \% \mathrm{Cl}$ & $p$ value & OR & $95 \% \mathrm{Cl}$ & $p$ value \\
\hline \multicolumn{7}{|l|}{ Pretransplant characteristics } \\
\hline Emergent heart transplantation* & 3.34 & $1.04-10.69$ & 0.04 & & & \\
\hline In ICU at the time of transplantation & 2.99 & $0.85-10.49$ & 0.08 & & & \\
\hline Waiting time, $d$ & 0.99 & $0.99-1.00$ & 0.07 & & & \\
\hline Acute liver failure (bilirubin $>30 \mu \mathrm{mol} / \mathrm{L}$ ) & 9.64 & $1.15-80.53$ & 0.03 & & & \\
\hline Low cardiac output syndrome & 2.97 & $0.96-9.06$ & 0.05 & & & \\
\hline Mean pulmonary arterial pressure, $\mathrm{mm} \mathrm{Hg}$ & 0.93 & $0.88-0.98$ & 0.01 & & & \\
\hline MELD-XI score & 1.16 & $1.02-1.31$ & 0.02 & & & \\
\hline \multicolumn{7}{|l|}{ Posttransplant characteristics } \\
\hline Cardiac arrest & 8.25 & $2.39-28.48$ & 0.001 & & & \\
\hline Maximum adrenaline dose, $\mu \mathrm{g} / \mathrm{kg}$ per min & 1.74 & $0.96-2.87$ & 0.12 & & & \\
\hline Inotrope duration & 0.91 & $0.82-1.00$ & 0.06 & & & \\
\hline Delayed initiation of VA-ECMO & 8.25 & $2.39-28.48$ & 0.001 & 5.11 & $1.08-24.22$ & 0.04 \\
\hline Central & 19.6 & $2.41-159.9$ & 0.005 & & & \\
\hline 24-h bleeding & 1.01 & $1.00-1.02$ & 0.05 & 1.00 & $1.00-1.01$ & 0.05 \\
\hline \multicolumn{7}{|c|}{$\begin{array}{l}\text { Note: Univariate testing was used as a prescreening method for inclusion of variables in the multivariable model. The highest threshold used was } p<0.25 \text {. } \mathrm{Cl}= \\
\text { confidence interval; ICU = intensive care unit; } \mathrm{MELD}-\mathrm{XI}=\text { Model for End-stage Liver Disease score excluding INR; } \mathrm{OR}=\text { odds ratio; } \mathrm{VA}-\mathrm{ECMO}=\text { venoarterial } \\
\text { extracorporeal membrane oxygenation. }\end{array}$} \\
\hline
\end{tabular}

A

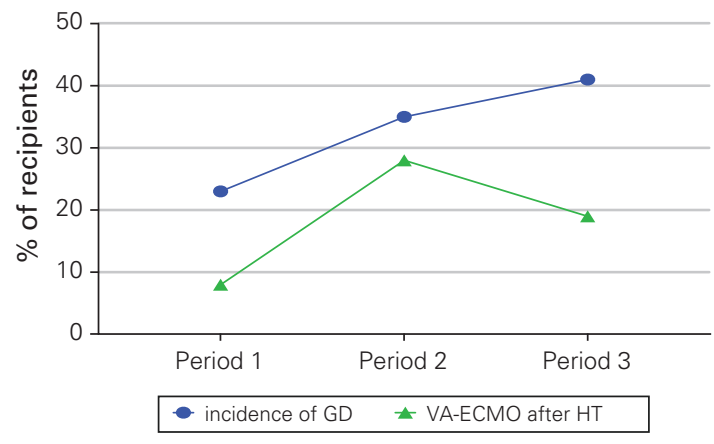

B

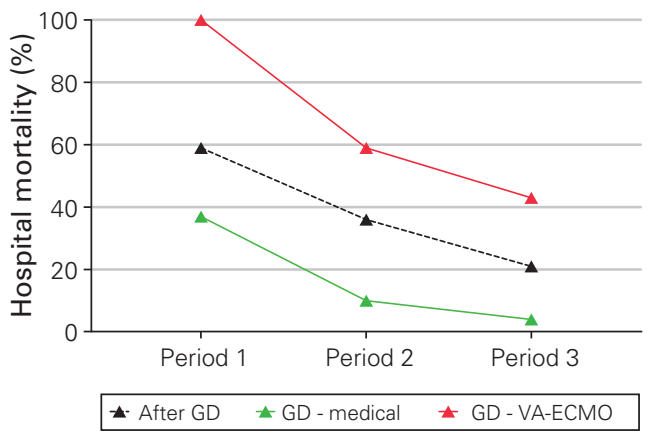

Fig. 4. (A) Incidence of GD and use of VA-ECMO after HT in the 3 study periods. (B) Hospital mortality in all HT patients with GD and in the subgroups of patients with GD treated medically or with VA-ECMO support across study periods. GD = graft dysfunction; $\mathrm{HT}$ = heart transplantation; $\mathrm{VA}-\mathrm{ECMO}=$ venoarterial extracorporeal membrane oxygenation.

age of recipients and donors increased significantly over time, with almost half of donors being older than 50 years in the third period (Appendix 1, Tables 4S and $5 \mathrm{~S})$.

The use of VA-ECMO increased substantially between periods 1 and 2 (from 9\% to 28\%) and decreased slightly in period 3 (19\% of all recipients in the corresponding period, $p=0.001$ ) (Figure 4A). The rates of early initiation among patients supported by VAECMO increased over time from $50 \%$ to $68 \%(p=0.01)$.

The overall in-hospital mortality in the entire cohort decreased from $59 \%$ to $21 \%(p=0.001)$ (Figure 4B), with a reduction of $52 \%$ in the VA-ECMO group and of $33 \%$ in the medically treated group between the first and third periods. Post-transplant survival increased over the study period (Figure 3D).

\section{Discussion}

In this study, we reviewed all of the adult heart recipients who met the new criteria for GD in 3 cardiac centres over a 10 -year period. Our main findings were as follows: the incidence of GD increased from $23 \%$ to $41 \%$ over the study period, the only factor associated with the use of VA-ECMO was the peak levels of serum lactates after HT, the hospital mortality rate decreased over time in patients treated with and without VA-ECMO, mortality among patients who required VA-ECMO remained high and delayed initiation of VA-ECMO and postoperative bleeding were strongly associated with mortality.

According to the 33rd Adult Heart Transplantation Report of the ISHLT, graft failure remains the leading cause of early death after HT. ${ }^{12}$ The definition of GD 
varies among studies, making intercentre comparisons and reports difficult to interpret. Several studies have defined GD as the need for mechanical circulatory support, which is only a part of the definition. Many efforts have been made to better standardize the criteria for diagnosing primary GD. ${ }^{2,11,13}$ The incidence of GD in our cohort is higher $(33 \%)$ than in previously published reports ( $4.4 \%$ to $24 \%$ ). There might be several reasons for this difference: the use of broader criteria to retrospectively define GD, the greater number of high-risk recipients $(27 \%$ had inotropic support and $27 \%$ were older than $60 \mathrm{yr}$ ) and the high rate of marginal donors (27\% were older than $50 \mathrm{yr}$ and $38 \%$ had a graft ischemia time exceeding $4 \mathrm{~h}$ ).

Peripheral or central VA-ECMO is probably the most appropriate form of mechanical support for GD after HT. 3,4,6,7, , 10,14-16 Its use in critically ill patients was introduced more than 15 years ago, and several retrospective studies have since suggested that VA-ECMO is superior to other mechanical support devices. ${ }^{7,16}$ One of the most important advantages of VA-ECMO is that it can provide rapid, reproducible and complete circulatory support. It is also available any time and can easily be initiated in the operating room or in the intensive care unit. When peripheral, VA-ECMO can be weaned progressively and removed without reopening the chest after graft recovery. For these reasons, VA-ECMO is the first-line mechanical support for cardiogenic shock in our institutions. ${ }^{17-20}$ We chose to use VA-ECMO as biventricular support even in isolated RV failure, which was the most frequent clinical presentation of GD in our series.

The management of GD changed slightly over the study period, with more frequent use of VA-ECMO for patients with circulatory failure over time. However, we did not use a specific algorithm for decision-making about initiation of VA-ECMO. The decision to support patients with VA-ECMO was made by the physicians and surgeons responsible for patient care. Among the 66 patients who had VA-ECMO after HT, 35 received it at the Rennes University Hospital, 18 at the IUCPQ and 13 at the MHI. This reflects a more liberal use of VA-ECMO at the Rennes University Hospital.

VA-ECMO complications occur frequently and are potentially life threatening. In our study, most of the patients had ECMO-related complications during mechanical support: $64 \%$ needed reoperation for bleeding or tamponade, 100\% required transfusions for bleeding and $11 \%$ had lower limb ischemia despite a homolateral reperfusion cannula. The main cause of death under VA-ECMO support was multiorgan failure (33\%), which is consistent with other studies.

Not surprisingly, survival of patients with GD requiring mechanical support after HT is lower. In a retrospective study of 51 patients with PGD, Marasco and colleagues used an interesting algorithm for the early initiation of mechanical circulatory support after HT. ${ }^{9}$ They described 15 patients who required various mechanical circulatory supports:
4 received VA-ECMO, 4 received right-sided support alone using a centrifugal pump, 3 received left-sided support alone using a pulsatile pump and 4 received biventricular support. Their study population had an in-hospital survival rate of $66 \%$, which is close to the $60 \%$ observed in our early-ECMO subgroup. In a study of 54 patients supported by VA-ECMO within the first 48 hours after HT, D'Alessandro and colleagues reported a $50 \%$ in-hospital mortality rate. ${ }^{3}$ Interestingly, the delay of initiation was short (4 h) and $76 \%$ of the patients of this study were supported before leaving the operating room. Unfortunately, there was no survival analysis based on the delay of initiation in their study.

Several studies have shown that prompt extracorporeal life support with VA-ECMO is asociated with better survival than conventional management in patients with severe ischemic cardiogenic shock or in-hospital cardiac arrest. ${ }^{17,21-24}$ The same observation has been made in patients with $\mathrm{RV}$ failure after LVAD implantation. In a comparison of patients who received a planned biventricular assist device and a delayed biventricular assist device, Fitzpatrick and colleagues showed superior survival until discharge in the planned group..$^{25}$

Although recipient and donor characteristics were similar between the early-ECMO and delayed-ECMO subgroups, patients who underwent early VA-ECMO implantation exhibited a greater number of recognizable risk factors for early GD after HT. This observation could be explained by a lower threshold for VA-ECMO initiation in such patients: we try to avoid reaching high doses of inotropes before VA-ECMO initiation, especially when the probability of GD is high. The higher survival among patients with early VA-ECMO initiation suggests that this approach should be promoted.

\section{Limitations}

The current analysis has the limitations of a retrospective study. The number of patients was limited, but our study is among the largest published to date and included patients from 3 institutions. Rapid identification of patients who develop GD after HT is somewhat challenging and requires a high index of suspicion. In the absence of standardized decisional algorithms for VA-ECMO initiation, it is likely that some differences in medical management existed between the study institutions and even between physicians within the same hospital. We cannot completely rule out the possibility that differences in medical management may have had an impact on the study outcomes. Furthermore, there were many confounding factors that were not included in the current analysis (e.g., operative techniques, quality of graft retrieval and graft preservation). Another limitation is the large spectrum of the clinical status of recipients before VA-ECMO initiation, ranging from the rescue situation during resuscitation for cardiac arrest to pre-emptive initiation of VA-ECMO after 1 or more attempts to wean patients off cardiopulmonary bypass. 


\section{Conclusion}

The incidence of GD increased over time in this study, and the need for VA-ECMO among patients with GD remained difficult to predict. The mortality rate decreased over the study period, but it remained high among patients who required VA-ECMO, especially in those with a delayed initiation of VA-ECMO.

Affiliations: From the Department of Cardiac Surgery, Montreal Heart Institute, Université de Montréal, Montréal, Que. (Noly, Hébert, Lamarche, Carrier); the Department of Cardiac Surgery, Quebec Heart and Lung Institute, Université Laval, Québec, Que. (Cortes, Voisine); and the Department of Thoracic and Cardiovascular Surgery, Rennes Hospital, University of Rennes 1, Rennes, France (Mauduit, Verhoye, Flécher).

Competing interests: None declared.

Contributors: P.-E. Noly, J.-P. Verhoye and P. Voisine conceived the study. P.-E. Noly, M. Hébert, J.R. Cortes, M. Mauduit and E. Flécher acquired the data, which P.-E. Noly, M. Hébert, Y. Lamarche, P. Voisine, E. Flécher and M. Carrier analyzed. P.-E. Noly and M. Hébert wrote the article, which all authors critically revised. All authors agreed to be accountable for all aspects of the work.

Content licence: This is an Open Access article distributed in accordance with the terms of the Creative Commons Attribution (CC BY-NC-ND 4.0) licence, which permits use, distribution and reproduction in any medium, provided that the original publication is properly cited, the use is noncommercial (i.e., research or educational use), and no modifications or adaptations are made. See: https://creativecommons.org/licenses/by-nc-nd/4.0/

\section{References}

1. Lund LH, Khush KK, Cherikh WS, et al. The Registry of the International Society for Heart and Lung Transplantation: Thirty-fourth Adult Heart Transplantation Report-2017; Focus Theme: Allograft ischemic time. 7 Heart Lung Transplant 2017;36:1037-46.

2. Kobashigawa J, Zuckermann A, Macdonald P, et al. Report from a consensus conference on primary graft dysfunction after cardiac transplantation. F Heart Lung Transplant 2014;33:327-40.

3. D'Alessandro C, Aubert S, Golmard JL, et al. Extra-corporeal membrane oxygenation temporary support for early graft failure after cardiac transplantation. Eur 7 Cardiothorac Surg 2010;37: 343-9.

4. D'Alessandro C, Golmard JL, Barreda E, et al. Predictive risk factors for primary graft failure requiring temporary extra-corporeal membrane oxygenation support after cardiac transplantation in adults. Eur 7 Cardiothorac Surg 2011;40:962-9.

5. Takeda K, Li B, Garan AR, et al. Improved outcomes from extracorporeal membrane oxygenation versus ventricular assist device temporary support of primary graft dysfunction in heart transplant. $\mathcal{F}$ Heart Lung Transplant 2017;36:650-6.

6. Kittleson MM, Patel JK, Moriguchi JD, et al. Heart transplant recipients supported with extracorporeal membrane oxygenation: outcomes from a single-center experience. 7 Heart Lung Transplant 2011;30:1250-6.

7. Leprince $\mathrm{P}$, Aubert S, Bonnet N, et al. Peripheral extracorporeal membrane oxygenation (ECMO) in patients with posttransplant cardiac graft failure. Transplant Proc 2005;37:2879-80.

8. Listijono DR, Watson A, Pye R, et al. Usefulness of extracorporeal membrane oxygenation for early cardiac allograft dysfunction. 7 Heart Lung Transplant 2011;30:783-9.
9. Marasco SF, Esmore DS, Negri J, et al. Early institution of mechanical support improves outcomes in primary cardiac allograft failure. $\mathcal{F}$ Heart Lung Transplant 2005;24:2037-42.

10. Marasco SF, Vale M, Pellegrino V, et al. Extracorporeal membrane oxygenation in primary graft failure after heart transplantation. Ann Thorac Surg 2010;90:1541-6.

11. Segovia J, Cosio MD, Barcelo JM, et al. RADIAL: a novel primary graft failure risk score in heart transplantation. 7 Heart Lung Transplant 2011;30:644-51.

12. Lund LH, Edwards LB, Dipchand AI, et al. The Registry of the International Society for Heart and Lung Transplantation: Thirtythird Adult Heart Transplantation Report-2016; Focus Theme: Primary Diagnostic Indications for Transplant. 7 Heart Lung Transplant 2016;35:1158-69.

13. Cosio Carmena MD, Gomez Bueno M, Almenar L, et al. Primary graft failure after heart transplantation: characteristics in a contemporary cohort and performance of the RADIAL risk score. 7 Heart Lung Transplant 2013;32:1187-95.

14. Chou NK, Chi NH, Ko WJ, et al. Extracorporeal membrane oxygenation for perioperative cardiac allograft failure. ASAIO $\mathrm{f}$ 2006;52:100-3.

15. Arpesella G, Loforte A, Mikus E, et al. Extracorporeal membrane oxygenation for primary allograft failure. Transplant Proc 2008;40:3596-7.

16. Taghavi S, Zuckermann A, Ankersmit J, et al. Extracorporeal membrane oxygenation is superior to right ventricular assist device for acute right ventricular failure after heart transplantation. Ann Thorac Surg 2004;78:1644-9.

17. Anselmi A, Flecher E, Corbineau H, et al. Survival and quality of life after extracorporeal life support for refractory cardiac arrest: a case series. 7 Thorac Cardiovasc Surg 2015;150:947-54.

18. Flecher E, Anselmi A, Corbineau H, et al. Current aspects of extracorporeal membrane oxygenation in a tertiary referral centre: determinants of survival at follow-up. Eur 7 Cardiothorac Surg 2014:46:665-71, discussion 671.

19. Le Pennec-Prigent S, Flecher E, Auffret V, et al. Effectiveness of extracorporeal life support for patients with cardiogenic shock due to intractable arrhythmic storm. Crit Care Med 2017;45:e281-9.

20. Muller G, Flecher E, Lebreton G, et al. The ENCOURAGE mortality risk score and analysis of long-term outcomes after VA-ECMO for acute myocardial infarction with cardiogenic shock. Intensive Care Med 2016;42:370-8.

21. Chen JS, Ko WJ, Yu HY, et al. Analysis of the outcome for patients experiencing myocardial infarction and cardiopulmonary resuscitation refractory to conventional therapies necessitating extracorporeal life support rescue. Crit Care Med 2006;34:950-7.

22. Chen YS, Lin JW, Yu HY, et al. Cardiopulmonary resuscitation with assisted extracorporeal life-support versus conventional cardiopulmonary resuscitation in adults with in-hospital cardiac arrest: an observational study and propensity analysis. Lancet 2008;372:554-61.

23. Combes A, Leprince P, Luyt CE, et al. Outcomes and long-term quality-of-life of patients supported by extracorporeal membrane oxygenation for refractory cardiogenic shock. Crit Care Med 2008; $36: 1404-11$

24. Sheu JJ, Tsai TH, Lee FY, et al. Early extracorporeal membrane oxygenator-assisted primary percutaneous coronary intervention improved 30-day clinical outcomes in patients with ST-segment elevation myocardial infarction complicated with profound cardiogenic shock. Crit Care Med 2010;38:1810-7.

25. Fitzpatrick JR III, Frederick JR, Hiesinger W, et al. Early planned institution of biventricular mechanical circulatory support results in improved outcomes compared with delayed conversion of a left ventricular assist device to a biventricular assist device. $\mathcal{F}$ Thorac Cardiovasc Surg 2009;137:971-7. 